\title{
Rationing in the Pediatric Intensive Care Unit-ethical or unethical?
}

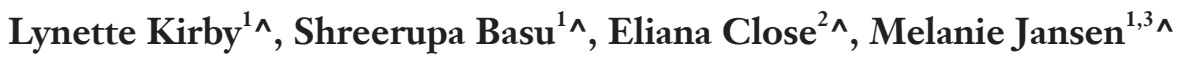 \\ ${ }^{1}$ Pediatric Intensive Care Unit, The Children's Hospital at Westmead, Sydney, NSW, Australia; ${ }^{2}$ Australian Centre for Health Law Research, Faculty of \\ Law, Queensland University of Technology, Brisbane, Queensland, Australia; ${ }^{3}$ Faculty of Medicine, University of Queensland, Queensland, Australia \\ Contributions: (I) Conception and design: All authors; (II) Administrative support: None; (III) Provision of study materials or patients: None; (IV) \\ Collection and assembly of data: None; (V) Data analysis and interpretation: None; (VI) Manuscript writing: All authors; (VII) Final approval of \\ manuscript: All authors. \\ Correspondence to: Lynette Kirby. Pediatric Intensive Care Unit, The Children's Hospital at Westmead, Locked Bag 4001, Westmead 2145, NSW, \\ Australia. Email: Lynette.kirby@health.nsw.gov.au.
}

\begin{abstract}
Rationing in health care is controversial, and even more so in pediatrics. Children are an inherently vulnerable group because they are reliant on their parents and caregivers to make decisions in their best interests and have no political voice. Historically, there has been general acceptance of the need to ration healthcare at a systems level, however there is controversy over whether healthcare professionals should be involved in rationing at the bedside. The COVID-19 pandemic has highlighted that bedside rationing is unavoidable, at least in times of extreme resource scarcity. Internationally, there has been significant ethical analysis and guideline development to guide intensive care rationing decisions in the event that resources are overwhelmed. This paper explores the principles underlying distributive justice in healthcare rationing and discusses how these were operationalized in ethical guidelines for the COVID-19 pandemic. In fact, rationing is unavoidable and occurs constantly in everyday nursing and medical ICU practice, often in mundane and uncontroversial ways. Some argue that these everyday decisions are not true rationing decisions, but resource allocation, or stewardship decisions. We argue there are no clear lines between resource allocation and rationing decisions, rather that they occur on a spectrum. These everyday rationing decisions are particularly susceptible to personal biases that are often implicit. Due to the subtle and constant nature of most everyday rationing decisions, specific guideline development will rarely be practical or appropriate. However, it is possible to develop other processes to improve decision making. There are a variety of strategies we recommend for this including, encouraging reflective practice; developing explicit frameworks that promote collaborative decision making; being transparent about resource allocation and rationing decisions with colleagues, patients, and families; and promoting a workplace culture of speaking up and accessing support in identifying and managing everyday rationing decisions.
\end{abstract}

Keywords: Rationing; bedside rationing; intensive care; pediatric intensive care

Submitted Oct 13, 2020. Accepted for publication Jan 29, 2021.

doi: $10.21037 / \mathrm{tp}-20-334$

View this article at: http://dx.doi.org/10.21037/tp-20-334

^ ORCID: Lynette Kirby, 0000-0003-4757-9024; Shreerupa Basu, 0000-0002-3177-627X; Eliana Close, 0000-0002-7359-3375; Melanie Jansen, 0000-0002-4609-9179. 


\section{Introduction}

Rationing in health care is controversial, and even more so when discussing rationing of healthcare for children. Children are an inherently vulnerable group because they are reliant on their parents and caregivers to make decisions in their best interests and have no political voice.

There is broad acceptance of the need to ration at a policy and systems level; however, there is ongoing reluctance to address bedside rationing as an inherent and inexorable task of clinicians $(1,2)$. The COVID-19 pandemic has brought explicit bedside rationing to the forefront of healthcare dialogue. Internationally, there has been significant ethical analysis and guideline development to guide intensive care rationing decisions in the event that health care resources are overwhelmed (3-7). Implicit in many of these guidelines is the idea that rationing decisions do not occur during every day clinical care, only when resources are in danger of being truly overwhelmed.

This paper intends to provide greater understanding of everyday rationing in the Pediatric Intensive Care Unit (PICU), using insights drawn from rationing frameworks developed for the COVID-19 pandemic. We use Ubel's broad definition of health care rationing, which includes any "implicit or explicit mechanism that allows people to go without beneficial services" (8). We argue that rationing in PICU is always unavoidable, and that there are in fact no clear lines between rationing decisions and resource allocation or stewardship decisions but rather they occur on a spectrum.

We begin with an overview of the theoretical basis for rationing in health care. We then outline how rationing frameworks during COVID-19 have operationalized these principles, drawing attention to the concept of a 'tipping point'-the point at which true resource scarcity renders rationing decisions unavoidable. Following this, we discuss rationing outside of the pandemic context. We canvas the evidence for when and how it occurs in both nursing and medical practice, and argue that rationing decisions are in fact unavoidable in everyday practice and we should develop explicit mechanisms to ensure that it is done ethically. We make several recommendations for improving rationing decisions in everyday practice. While our perspective is from the pediatric setting, the evidence discussion, and recommendations are generalisable to adult settings. We provide three clinical vignettes illustrating everyday bedside rationing decisions, that we invite the reader to contemplate as they read through our paper.

\section{Case 1}

The PICU is at capacity for the night shift and there is the minimum number of nursing staff rostered on after several sick calls with high patient acuity. A large proportion of the nurses are new graduates or have less than two years of PICU experience. The nursing team leader had difficulties allocating staff for the shift, particularly which nurse to place with a 2-year-old well-known PICU patient whose family have behaved aggressively towards each other and staff during the day. She decides to allocate a more experienced nurse to this patient as some of the junior staff had been upset after caring for this patient. A less experienced nurse, who is not yet ventilator trained, is allocated to a 6-monthold post-operative cardiac patient who was extubated late in the afternoon. Due to the high proportion of junior nurses, the 'float' nurses are particularly busy. A few hours into the shift, the post op cardiac patient deteriorates slowly, developing a low cardiac output state that progresses to cardiorespiratory arrest requiring $\mathrm{CPR}$ and reintubation.

On this same shift, a 13 -year-old boy with a severe traumatic brain injury is intubated, ventilated, muscle relaxed, sedated, and has an external ventricular drain. He requires second hourly log rolls for pressure area care and is being cared for in an isolation room by a senior nurse. The patient next door is an unsettled 6-week-old baby girl with bronchiolitis on humidified high flow nasal cannula, cared for by the new graduate nurse who is unable to relieve the senior nurse next door. The trauma patient develops raised intracranial pressure and requires an urgent CT brain. Due to short staffing, the patient has only been log rolled twice in the shift, and the nurse has only been able to attend mouth and eye care once. The nurse caring for the baby with bronchiolitis voices her frustration that she is not able to help the nurse next door or expand her knowledge and skills of trauma patients. Neither nurse gets a break until late in the shift.

\section{Case 2}

As the PICU staff specialist on service, you are responsible for the care of 12 patients. Each morning, approximately 2 hours are available for the daily ward round during which patients are reviewed and plans made for the day. In bed 4 is a 3 -year-old girl who is progressing well post liver transplant. Her mother is present during the ward round each morning. She is a general practitioner who attended your same medical college and has pertinent and 
interesting questions regarding her daughter's care. She is pleasant and engaging and during ward rounds, you spend 20 minutes at the bedside discussing her child's care as well as some anecdotes from college. In bed 9 is an 8 -year-old boy with a severe exacerbation of asthma on a background of poor compliance and known household smoking. He is improving very slowly with non-invasive respiratory support and asthma therapy. His parents are not present during morning rounds. You review him efficiently, with plans to review him again later and to discuss his current and future treatment with a parent when they arrive. Unfortunately, the day becomes busy and you fail to meet with his parents. In bed 12 is a 15 -month-old girl with a neurodegenerative illness who has a lower respiratory tract infection requiring some increased support from her usual baseline but is clinically stable. Her father can be hostile and demanding during clinical interactions, often requiring long conversations at the bedside to address all his concerns. Given your ward round has already run overtime, you review her observations prior to entering the room and leave the junior staff to assess her for the day and ask them to feed back to you any concerns.

\section{Case 3}

There is one bed available in PICU. Within minutes of each other, two children present to the Emergency Department (ED) — a 5-year-old with severe sepsis and a 14-year-old with serious injuries following a motor vehicle collision. Both are critically unwell and require PICU admission. The PICU nursing team leader and staff specialist are reviewing the current inpatients to see who might be suitable for early discharge to the ward. A 6-week-old baby with bronchiolitis was extubated to high flow nasal cannula three hours ago. Usual practice is to observe for 6 hours before discharge to the ward. The baby has mild-moderate work of breathing but you think he is likely to settle on his current support and is at low risk of deterioration. His mother is at the bedside. She is highly anxious and has had many questions throughout the admission, including asking at the beginning of each shift how experienced the nurses and doctors looking after her baby are. The other option is a 4-year-old who has been admitted for observation following surgical removal of a brain tumour. He was admitted 12 hours ago for 24 hours of neurological observation as the neurosurgeon is concerned about a high risk of bleeding. His father has been at the bedside throughout the afternoon, has been pleasant to the bedside nurses and has frequently thanked people for their efforts in looking after his son.

\section{Healthcare rationing-theoretical background}

A rationing decision occurs when a patient is denied healthcare from which they could benefit. Rationing decisions are distinct from decisions to withhold or withdraw therapy based on a patient's best interests. Deeming a treatment ineffective or not beneficial and then withholding or withdrawing that treatment is not a rationing decision, but rather a decision made on the basis of the patient's best interests. Rationing of healthcare is unavoidable because all resources are finite. Efficiency can be maximised, budgets can be increased, but healthcare needs are essentially limitless and so rationing will always occur (9).

Some argue that rationing should occur solely at a political or hospital executive level (10-12). Others concede that while healthcare professionals should have a role in rationing decisions, it ought only to be through involvement in policy and guideline development rather than making decisions at the bedside (13). The wording of medical and nursing codes of ethics reflects this discomfort with bedside rationing. The American Medical Association's code of ethics states, "The relationship between patient and physician is based on trust and gives rise to physicians' ethical obligations to place patients' welfare above their own self-interest and above obligations to other groups, and to advocate for their patients' welfare." (14). In the Nursing Code of Ethics, "The nurse advocates for equity and social justice in resource allocation, access to health care and other social and economic services."; and direction is given for researchers and educators to include "references to buman rights, equity, justice, and solidarity as the basis for access to care" in curriculums, but no reference is made to direct involvement in rationing decisions (15).

\section{The CovID-19 rationing context}

The COVID-19 pandemic has made bedside rationing visible through highlighting its inevitability in the pandemic context. Those in Italy describe the injustices of a first come, first served system of rationing, and the extreme stress placed on frontline clinicians (16). Since the pandemic began, there have been unprecedented numbers of publications exploring the ethical implications of rationing intensive care beds, and a huge amount of scholarly work has gone into developing triage tools (17-23). Many 
emphasise the longstanding focus in the rationing literature on procedural justice. The most well-known approach to procedural justice in healthcare is the framework described by Norman Daniels and James Sabin (9). They begin with the premise that no consensus on the ethical principles upon which to base prioritisation and allocation of resources exists, despite concerted effort in the health economics and ethics spheres. In the absence of consensus on what constitutes substantive justice, Daniels and Sabin argue that it is important to focus on the process for decision-making. This means that even though reasonable and fair minded people may disagree on the priorities set in a particular case, they can be reassured that the decision is legitimate by virtue of it having been made through a fair process. To establish legitimacy, the decision making process must satisfy conditions that hold decision makers "accountable for reasonableness" $(24,25)$.

Daniels proposes four conditions for a fair resource allocation process:

(I) Publicity: the rationale for all limit-setting decisions must be publicly accessible;

(II) Relevance: the rationales for decisions must aim to provide a reasonable explanation for how the decision makers are providing cost effective care to the population;

(III) Appeals: there must be a mechanism to challenge and revise rationing decisions;

(IV) Enforcement: regulation of the process ensures that the first three conditions are satisfied.

Many COVID-19 intensive care rationing guidelines have attempted to fulfil these four conditions. Most recommend decision-making by a 'triage team' that is at least somewhat separate to bedside clinicians (21-23). Suggested membership of this team is variable and dependent on each institution's needs and resources. Many also recommend a committee to review triage decisions and to manage appeal mechanisms, within the practical boundaries of what is reasonable in a rapidly evolving pandemic. These guidelines outline explicit criteria for ICU admission, provide tools to help determine the clinical priority of patients, and suggest 'tie breakers' for use in decisions between patients who are both eligible and of equal clinical priority; outlining what factors are to be considered for bedside rationing decisions. Organisations fulfilling the publicity condition have made their processes publicly accessible, for example on the institutional website, or published in open access journals (21).

Of note, most of these rationing guidelines define criteria for a 'tipping point' or 'switch'. This is the point where an organisation recognises that they cannot provide usual access to care due to the increased demand on resources. The organisation then 'switches' to an approach where the most efficient use of resources for their community becomes an explicit part of decision making about who receives intensive care $(22,23,26)$. Defining a tipping point to activate explicit pandemic rationing frameworks is of practical importance because these decision-making processes are labour intensive and complex. Whilst this is practically important, the idea of a 'switch' or 'tipping point' implies that bedside rationing does not occur outside of pandemic conditions. Yet, as we argue in the next section, bedside rationing decisions are made on a daily basis during the delivery of usual care. Principles of decision making elucidated in the pandemic context can be incorporated into our everyday practice to make the delivery of all healthcare fairer and more just.

\section{Rationing—only in extraordinary times?}

The acceptance of bedside rationing during the pandemic is driven by the perception of necessity in extraordinary times, making difficult decisions more palatable. The idea that bedside rationing happens in the everyday context remains anathema to many. The key objection is that it undermines the clinician-patient relationship, requiring clinicians to fulfil untenable dual roles of caregiver to an individual patient and rationer of community resources. Some argue that doctors must be solely patient advocates, and zealously defend their patients' interests even against the interests of society as a whole $(10,27)$. Clinicians tend to characterise allocative decisions as clinical ones (28) demonstrating variable willingness to acknowledge rationing at the bedside $(29,30)$.

In fact, bedside rationing is unavoidable. Truog et al. argue that rationing occurs constantly in the ICU, often in mundane ways such as deciding which staff members to allocate to care for which patients (as illustrated in case vignette 1), or whether urgent but elective surgical cases be cancelled to provide moderately unwell patients the benefit of intensive care monitoring (31). Rationing has also been described in the nursing literature. Nursing time spent on different care tasks and interactions with patients and their families are all rationing decisions made on a daily basis (32-35). Time spent with others (as illustrated in case vignette 2), care left incomplete and omissions of care (as illustrated in case vignette 1), are all acts of bedside rationing. Historically, this is a largely ignored phenomenon, particularly in nursing (34). 
Some would argue that everyday decisions like this are not rationing decisions, but resource allocation, or good stewardship of resources (36). They assert that this is distinct from rationing because healthcare is not denied, and instead resources are used more efficiently, meaning that no one misses out. We, and others, argue that there is no clear ethical line between 'rationing' and 'resource allocation' decisions, but rather they are a continuum (36-38). All resource allocation decisions involve the weighing of different patients' needs and an assessment of the (always) finite resources available to 'spend'. How significant does the effect of not allocating a certain resource have to be before considering it rationing? Which benefits or harms should we take into account when prioritising? The focus should be on the decision making process and how the priorities were weighed, rather than whether the decision is a 'true' rationing decision or not.

Discomfort raised by bedside rationing should not lead us to deny its occurrence. Consistent with Daniels' approach to procedural justice, Newdick et al. (in relation to the COVID-19 pandemic), point out that while it is impossible to ensure an absolute consistency of outcomes, from both a legal and an ethical perspective it is critical to foster consistent procedures to ensure decisions are legitimate (37). We can use some of the lessons learned in developing bedside rationing during COVID-19 and apply them to the spectrum of everyday rationing decisions in PICU.

\section{The ethics of 'everyday' rationing in PICU}

The pediatric context has several unique factors that make bedside rationing difficult. Historically, in developed health care systems, there has been an overwhelming sense of the 'rule of rescue'. This favours beneficence over consideration of distributive justice in maximising outcomes (39). The narrative of rescue is pervasive in pediatric practice, heightened by the sense that a child's death or serious illness always seems outside the natural order of things (40). High profile legal cases involving disputes over life-sustaining or experimental treatments avoid addressing resource rationing as a basis for decision making (41). Wilkinson and Savulescu point out that best interests or other ethical arguments may be applied to mask rationing decisions in these cases (42).

The taxonomy designed for intensive care clinicians by Truog et al. (31) suggests three primary types of rationing decisions:

(I) Rationing decisions based on external constraints (e.g., 1 bed available for 5 patients who would

\section{benefit);}

(II) Rationing decisions based on guidelines;

(III) Rationing decisions based on clinical judgement.

This taxonomy directs clinicians to consider the underlying ethical justification for rationing decisions and encourages awareness that they are susceptible to bias, as each type involves some degree of clinical discretion. It is asserted that the decisions based on clinical judgement are the most vulnerable to implicit bias and therefore are the most ethically problematic, as they may lack transparency and consistency and can occur without the clinician's, patient's or family's knowledge (31).

\section{Vulnerability of clinical judgment}

Rationing decisions made by clinical judgement are particularly susceptible to personal biases. Empirical evidence confirms that bedside rationing decisions by clinical judgment can be influenced by implicit bias $(43,44)$, such as ageism, racism or disability discrimination (45-47), though some argue this is justified (48). Bias may occur in other aspects of clinical judgement, for example, Wilkinson and Truog describe the 'roster lottery'-where end of life decisions differ significantly depending on which doctor is rostered on (49). Individual decisions can also be affected by a patient's characteristics, including their likeability (50) (as illustrated in case vignette 3). They can also be affected by a healthcare professional's background, including religion, culture and country of origin, personality, and medical specialty (51-55). A study of adult ICU practices across several countries found that medical practitioners were more likely to issue a 'do not attempt resuscitation' order on patients who were perceived to have a poorer job status (56). Some professionals also endorse limiting treatment on the basis of a patient's drug or alcohol addiction (57). Papastavrou et al.'s systematic review of quantitative studies of nursing care rationing identifies three core facets: (I) the nursing workload and professional environment; (II) rationing contributing to outcomes and quality and safety matters; and (III) the ethical and philosophical viewpoint of rationing (33). They report definite trends of rationing focused on biomedical and clinical tasks (such as eye and mouth care) more so than human need factors (interactions with patients and families).

\section{Combatting implicit bias: making clinical judgement more ethical}

Variation in clinical decision making is not always 
problematic (49). Patients' and families' need and preferences for care differ and should be responded to appropriately. It is also completely normal for clinicians to 'like' some patients more than others and enjoy interacting more with some families. It becomes problematic when ethically irrelevant factors drive variation in clinical care. While it is not practicable to develop rationing guidelines that are as explicit and specific as COVID rationing guidelines, the principles of procedural justice can guide us to develop strategies to protect against unjustified variation in care.

The most important step is for clinicians and departmental leadership to recognise this vulnerability. This creates opportunities to develop guidelines and processes to improve decision making quality. In addition, as demonstrated with the ICU triage tools for COVID-19, guidelines for decision making can help to decrease clinician moral distress associated with bedside rationing decisions. Due to the subtle and constant nature of most everyday rationing decisions, specific guideline development will rarely be practical or appropriate. However, it is possible to develop other processes to improve decision making. For example, in their systematic review of nursing rationing, Papastavrou et al. propose increased recognition and validated care omission reporting to minimise the threat to patient and clinician safety (34). This would also likely result in decreasing staff moral distress as there are explicit pathways through which to seek real time support in rationing care. Other strategies include education and training, promoting inclusivity and cultural curiosity, mentorship and sponsorship, and habitual, incremental action to promote genuine lasting change $(58,59)$.

Building cultural safety-awareness, and openness for psychologically safe discussion of possible biases will also protect day to day decision making from bias. Staff education to build awareness and encourage reflection of personal biases may be valuable. Tools such as the Harvard Implicit Association Test (IAT), may be a helpful resource for education $(60,61)$. Once recognition of rationing is established, explicit communication of the rationale for these decisions can become part of everyday clinical discussions. International studies concur that explicitness of decision making rationales is vital for legitimacy $(32,34,61)$. This also allows for retrospective review of decisions, which further improves quality and is consistent with the general principles of procedural justice, including publicity and appeals, in healthcare rationing.

\section{Recommendations for improving everyday rationing in PICU}

(I) Recognise the reality of everyday bedside rationing;

(II) Encourage reflective clinician practice. For example:

(i) Identify and \& interrogate your own and your team's implicit biases (e.g., using tools including the Harvard Implicit Association Test and by practising cultural safety);

(ii) Incorporate questions aimed at uncovering implicit bias (examples below) into operational meetings (e.g., morbidity \& mortality and casebased discussions).

(III) Where possible develop explicit frameworks that promote collaborative (rather than individual) decision-making;

(IV) Consider resource allocation when drafting all local practice guidelines and policies;

(V) Be transparent with colleagues, patients, and families when making rationing decisions;

(VI) Promote a workplace culture where people are encouraged to speak up if concerned about injustice and respectfully challenge possible bias;

(VII) Develop processes for staff to access support in identifying and managing everyday rationing decisions.

\section{Questions to target implicit bias}

(I) Does this patient or family have characteristics different to me (gender/culture/language/religion/ socio-economic status/lifestyle choices)?

(II) What is my emotional response to this patient?

(III) Do I like/dislike something about this patient or their family?

(IV) Do I feel conflicted about an aspect of this patient's care?

(V) Is there a power imbalance here and how does it impact my approach to this patient or their family?

(VI) Am I finding it difficult to communicate with this patient or family?

(VII) Should I discuss this with my team?

\section{Conclusions}

The COVID-19 pandemic has brought the ethics of bedside rationing to the forefront of healthcare discourse. While it is now widely accepted that rationing is unavoidable during a pandemic, many feel uncomfortable with bedside 
rationing in the context of 'everyday' care. Rationing is unavoidable and therefore we must do it consciously and do it well. The principles of procedural justice can be applied to everyday rationing decisions and be used to develop more robust decision making processes that are less vulnerable to implicit bias. We recommend that PICUs recognise the ubiquity of rationing; develop guidelines and processes for decision making where appropriate; and provide education that promotes reflection, open communication, and explicit discussion of rationing decisions in everyday care.

\section{Acknowledgments}

Funding: None.

\section{Footnote}

Provenance and Peer Review: This article was commissioned by the Guest Editors (Jan Hau Lee, Vijay Srinivasan, and Debbie Long) for the series "Pediatric Critical Care" published in Translational Pediatrics. The article has undergone external peer review.

Peer Review File: Available at http://dx.doi.org/10.21037/tp20-334

Conflicts of Interest: All authors have completed the ICMJE uniform disclosure form (available at http://dx.doi. org/10.21037/tp-20-334). The series "Pediatric Critical Care" was commissioned by the editorial office without any funding or sponsorship. The authors have no other conflicts of interest to declare.

Ethical Statement: The authors are accountable for all aspects of the work in ensuring that questions related to the accuracy or integrity of any part of the work are appropriately investigated and resolved.

Open Access Statement: This is an Open Access article distributed in accordance with the Creative Commons Attribution-NonCommercial-NoDerivs 4.0 International License (CC BY-NC-ND 4.0), which permits the noncommercial replication and distribution of the article with the strict proviso that no changes or edits are made and the original work is properly cited (including links to both the formal publication through the relevant DOI and the license). See: https://creativecommons.org/licenses/by-nc-nd/4.0/.

\section{References}

1. Close E, White BP, Willmott L, et al. Doctors' perceptions of how resource limitations relate to futility in end-oflife decision making: a qualitative analysis. J Med Ethics 2019;45:373-9.

2. Hurst SA, Danis M. A framework for rationing by clinical judgment. Kennedy Inst Ethics J 2007;17:247-66.

3. Antommaria AH, Sweney J, Poss WB. Pediatr Crit Care Med triaging pediatric critical care resources during a pandemic: ethical and medical considerations. Pediatric Crit Care Med 2010;11:396-400.

4. Kirby J. Enhancing the fairness of pandemic critical care triage. J Med Ethics 2010;36:758-61.

5. Jansen M, Irving H, Gillam L, et al. Ethical considerations for pediatrics during the COVID-19 pandemic:

A discussion paper from the Australian Pediatric Clinical Ethics Collaboration. J Paediatr Child Health 2020;56:847-51.

6. Kanter RK, Cooper A. Mass critical care: pediatric considerations in extending and rationing care in public health emergencies. Disaster Med Public Health Prep 2009;3 Suppl 2:S166-71.

7. Scheunemann LP, White DB. The Ethics and Reality of Rationing in Medicine. Chest 2011;140:1625-32.

8. Ubel P. Pricing Life: Why It's Time for Health Care Rationing. Massachusetts: Institute of Technology, 2000.

9. Daniels N, Sabin J. Setting Limits Fairly: Can We Learn to Share Medical Resources? New York: Oxford University Press, 2002.

10. Levinsky NG. The Doctor's Master. N Engl J Med 1984;311:1573-5.

11. Askin WJ. Bedside rationing. CMAJ 2002;166:711.

12. Schafer A. Bedside rationing by physicians: the case against. Healthc Pap 2001;2:45-52; discussion 69-75.

13. Capozzi JD, Rhodes R, Cornwall R. Bedside Rationing. J Bone Joint Surg Am 2002;84:1279-81.

14. The American Medical Association. Code of Medical Ethics. 2004. Available online: https://ama.com.au/system/ tdf/documents/AMA\%20Code\%20of\%20Ethics\%20 2004.\%20Editorially\%20Revised\%202006.\%20 Revised\%202016.pdf? file $=1 \&$ type $=$ node $\&$ id $=46014$

15. International Council of Nurses. The ICN Code of Ethics for Nurses. 2012. Available online: http://www.icn.ch/ who-we-are/code-of-ethics-for-nurses/

16. Rosenbaum L. Facing Covid-19 in Italy-Ethics, Logistics, and Therapeutics on the Epidemic's Front line. N Engl J Med 2020;382:1873-5. 
17. WHO. Ethics and COVID-19: resource allocation and priority setting. 2020. Available online: https://www. who.int/ethics/publications/ethics-covid-19-resourceallocation.pdf?ua $=1$

18. Antommaria AH. Ventilator Triage Policies During the COVID-19 Pandemic at U.S. Hospitals Associated With Members of the Association of Bioethics Program Directors. Ann Intern Med 2020;173:188-94.

19. Emanuel EJ, Persad G, Upshur R, et al. Fair Allocation of Scarce Medical Resources in the Time of Covid-19. N Engl J Med 2020;382:2049-55.

20. Joebges S, Biller-Andorno N. Ethics Guidelines On COVID-19 Triage-An Emerging International Consensus. Critical Care (London, England) 2020;24:201.

21. White DB, Lo B. A Framework for Rationing Ventilators and Critical Care Beds During the COVID-19 Pandemic. JAMA 2020;323:1773-4.

22. Dawson A, Isaacs D, Jansen M, et al. An ethics framework for making resource allocation decisions within clinical care: Responding to COVID-19. J Bioeth Inq 2020;17:749-55.

23. Warrillow S, Austin D, Cheung W, et al. ANZICS guiding principles for complex decision making during the COVID-19 pandemic. Crit Care Resusc 2020;22:98-102.

24. Daniels N. Just health care. New York: Cambridge University Press, 1985.

25. Daniels N. Symposium on the Rationing of Health Care: 2 Rationing Medical Care - A Philosopher's Perspective on Outcomes and Process. Economics and Philosophy 1998; 14:27-50.

26. Hurford JE. The BMA COVID-19 ethical guidance: a legal analysis. New Bioeth 2020;26:176-89.

27. Wyller V. Give to the doctor what is due to the doctor! Why "fair rationing at the bedside" is impossible. In: Danis M, Hurst S, Fleck L, editors. Fair Resource Allocation and Rationing at the Bedside. New York: Oxford University Press, 2014:253-62.

28. Strech D, Synofzik M, Marckmann G. How Physicians Allocate Scarce Resources at the Bedside: A Systematic Review of Qualitative Studies. J Med Philos 2008;33:80.

29. Syrett K. Law, Legitimacy, and the Rationing of Health Care: A Contextual and Comparative Perspective. Cambridge: Cambridge University Press, 2007.

30. Strech D. Are Physicians Willing to Ration Health Care? Conflicting Findings in A Systematic Review of Survey Research. Health Policy 2009;90:113-24.

31. Truog RD, Brock DW, Cook DJ, et al. Rationing in the intensive care unit*. Crit Care Med 2006;34:958-63.
32. Schubert M, Glass T, Clarke S, et al. Rationing of nursing care and its relationship to patient outcomes: the Swiss extension of the International Hospital Outcomes Study. Int J Qual Health Care 2008;20:227-37.

33. Papastavrou E, Andreou P, Efstathiou G. Rationing of nursing care and nurse-patient outcomes: a systematic review of quantitative studies. Int J Health Plann Manage 2014;29:3-25.

34. Papastavrou E, Andreou P, Tsangari H, et al. Rationing of nursing care within professional environmental constraints: a correlational study. Clin Nurs Res 2014;23:314-35.

35. Scott PA, Harvey C, Felmann H, et al. Resource allocation and rationing in nursing care: A discussion paper. Nurs Ethics 2019;26:1528-39.

36. Fleck LM. Choosing wisely is parsimonious care just rationing? Camb Q Healthc Ethics 2016;25:366-76.

37. Newdick C, Sheehan M, Dunn M. Tragic choices in intensive care during the COVID-19 pandemic: on fairness, consistency and community. J Med Ethics 2020;46:646-51.

38. Ubel PA, Goold S. Recognizing bedside rationing: Clear cases and tough calls. Ann Intern Med 1997;126:74-80.

39. Lawson A. Rationing in intensive care. J Intensive Care 2009;10:5-7.

40. Robinson $W$. The narrative of rescue in pediatric practice. In: Charon R, Montello M. Stories Matter: The Role of Narrative in Medical Ethics. New York: Routledge, 2002.

41. Close E, Willmott L, White BP. Charlie Gard: in defence of the law. J Med Ethics 2018;44:476-80.

42. Wilkinson D, Savalescu J. Ethics, Conflict and Medical Treatment for Children 1st Edition From disagreement to dissensus. London: Elsevier, 2018.

43. Chapman EN, Kaatz A, Carnes M. Physicians and implicit bias: how doctors may unwittingly perpetuate health care disparities. J Gen Intern Med 2013;28:1504-10.

44. FitzGerald C, Hurst S. Implicit bias in healthcare professionals: a systematic review. BMC Med Ethics 2017;18:19.

45. Leung S, Logiudice D, Schwarz J, et al. Hospital doctors' attitudes towards older people. Intern Med J 2011;41:308-14.

46. van Ryn M, Fu S. Paved with good intentions: Do Public Health and Human Service Providers Contribute to Racial/Ethnic Disparities in Health? Am J Public Health 2003;93:248-55.

47. Grady M, Edgar T. Racial Disparities in Healthcare: Highlights from Focus Group Findings. In: Smedley B, Stith A, Nelson A, editors. Unequal Treatment: 
Confronting Racial and Ethnic Disparities in Health Care. Washington (DC): National Academies Press (US), 2003.

48. Wilkinson D, Savulescu J. Disability, discrimination and death: is it justified to ration life saving treatment for disabled newborn infants?. Monash Bioeth Rev 2014;32:43-62.

49. Wilkinson DJ, Truog R. The luck of the draw: physicianrelated variability in end-of-life decision-making in intensive care. Intensive Care Med 2013;39:1128-32.

50. De Ruddere L, Goubert L, Prkachin K, et al. When you dislike patients, pain is taken less seriously. Pain 2011;152:2342-7.

51. Hinkka H, Kosunen E, Metsänoja R, et al. Factors affecting physicians' decisions to forgo life-sustaining treatments in terminal care. J Med Ethics 2002;28:109-14.

52. Frost DW, Cook DJ, Heyland DK, et al. Patient and healthcare professional factors influencing end-of-life decision-making during critical illness: A systematic review. Crit Care Med 2011;39:1174-89.

53. Ball CG, Navsaria P, Kirkpatrick AW, et al. The impact of country and culture on end-of-life care for injured patients: Results from an international survey. J Trauma 2010;69:1323-33; discussion 1333-4.

Cite this article as: Kirby L, Basu S, Close E, Jansen M. Rationing in the Pediatric Intensive Care Unit-ethical or unethical? Transl Pediatr 2021;10(10):2836-2844. doi: $10.21037 /$ tp-20-334
54. Poulton B, Ridley S, Mackenzie-Ross R, et al. Variation in end-of-life decision making between critical care consultants. Anaesthesia 2005;60:1101-5.

55. Parker MH, Cartwright C, Williams G. Impact of specialty on attitudes of Australian medical practitioners to end-oflife decisions. Med J Aust 2008;188:450-6.

56. Guyatt G, Cook D, Weaver B, et al. Influence of perceived functional and employment status on cardiopulmonary resuscitation directives. J Crit Care 2003;18:133-41.

57. Neuberger J. Public and professional attitudes to transplanting alcoholic patients. Liver Transpl 2007;13:S65-8.

58. Byrne A, Tanesini A. Instilling new habits: addressing implicit bias in healthcare professionals. Adv Health Sci Educ Theory Pract 2015;20:1255-62.

59. Marcelin JR, Siraj DS, Victor R, et al. The Impact of Unconscious Bias in Healthcare: How to Recognize and Mitigate It. J Infect Dis 2019;220:S62-73.

60. Greenwald AG, McGhee D, Schwartz J. Measuring individual differences in implicit cognition: the implicit association test. J Pers Soc Psychol 1998;74:1464-80.

61. Rooddehghan Z, Yekta Z, Nasrabadi A. Ethics of rationing of nursing care. Nurs Ethics 2018;25:591-600. 